\title{
Study of charmed strange baryons at Belle
}

\author{
Yuji Kato*i \\ Nagoya University \\ E-mail: kato@hepl.phys.nagoya-u.ac.jp
}

We report results of a study of charmed strange baryons. The analysis is performed using a 980 $\mathrm{fb}^{-1}$ data sample collected with the Belle detector at the KEKB asymmetric-energy $e^{+} e^{-}$collider. We search for two excited charmed strange baryons, $\Xi_{c}(3055)^{+}$and $\Xi_{c}(3123)^{+}$with $\Lambda_{c}^{+} K^{-} \pi^{+}$ final states through intermediate $\Sigma_{c}^{++}(2455)$ or $\Sigma_{c}^{++}(2520)$ resonances. The $\Xi_{c}(3055)^{+}$signal is observed with a significance of 6.6 standard deviations including systematic uncertainty, while no signature of the $\Xi_{c}(3123)^{+}$is seen. We also study $\Lambda D^{+(0)}$ final state. We observe decays of $\Xi_{c}(3055)^{+(0)}$ and $\Xi_{c}(3080)^{+}$into $\Lambda D^{+(0)}$. This is the first observation of the $\Xi_{c}(3055)^{0}$.

XV International Conference on Hadron Spectroscopy-Hadron 2013

4-8 November 2013

Nara, Japan

* Speaker.

${ }^{\dagger}$ for the Belle collaboration. 


\section{Introduction}

In recent years, there has been much progress in the experimental study of the charmed baryon spectroscopy mainly by Belle and BaBar experiments. In the charmed strange baryon sector, a number of excited states $\left(\Xi_{c}^{*}\right)$ has been observed. The Belle collaboration reported evidence of two excited states, $\Xi_{c}(2980)$ and $\Xi_{c}(3080)$, in the $\Lambda_{c}^{+} K^{-} \pi^{+}$and $\Lambda_{c}^{+} K_{S}^{0} \pi^{-}$final states [1]. These states are confirmed by $\mathrm{BaBar}$ later [2]. In the same paper, $\mathrm{BaB}$ ar also claimed evidence of two resonances, $\Xi_{c}(3055)^{+}$and $\Xi_{c}(3123)^{+}$, through intermediate $\Sigma_{c}(2455)^{++} K^{-}$and $\Sigma_{c}(2520)^{++} K^{-}$ final states. Independent search of these two states is necessary to confirm the existences. Among a number of possible decay modes of the charmed strange baryons, the $\Lambda D^{+(0)}$ mode is not studied well.

In this paper, we report the studies of charmed strange baryons in the $\Lambda_{c}^{+} K^{-} \pi^{+}$and $\Lambda D^{+(0)}$ final states using a data sample with an integrated luminosity of $980 \mathrm{fb}^{-1}$ collected with the Belle detector [3] at the KEKB asymmetric-energy $e^{+} e^{-}$collider [4]. All the results are preliminary.

\section{Event selection}

The $\Lambda_{c}^{+}$candidates are reconstructed via its decay to $p K^{-} \pi^{+}$and $p K_{S}^{0}[5]$. The $D^{+}$candidates are reconstructed via its decay to $K^{-} \pi^{+} \pi^{+}$. The $D^{0}$ candidates are reconstructed via its decays to $K^{-} \pi^{+}, K^{-} \pi^{+} \pi^{+} \pi^{-}$and $K^{-} \pi^{+} \pi^{0}$. The charged proton, kaon, and pion are required to have a point of closest approach to the interaction point that is within $0.2 \mathrm{~cm}$ in the transverse $(r-\phi)$ direction and within $2 \mathrm{~cm}$ along the $z$-axis. (The $z$-axis is opposite the positron beam direction.) For each track, the likelihood values $\mathscr{L}_{p}, \mathscr{L}_{K}$, and $\mathscr{L}_{\pi}$ are provided for the assumption of proton, kaon and pion, respectively. The likelihood ratio is defined as $\mathscr{L}(i: j)=\mathscr{L}_{i} /\left(\mathscr{L}_{i}+\mathscr{L}_{j}\right)$ and a track is identified as a proton if the likelihood ratios $\mathscr{L}(p: \pi)$ and $\mathscr{L}(p: K)$ are greater than 0.6. A track is identified as a kaon if the likelihood ratios $\mathscr{L}(K: \pi)$ and $\mathscr{L}(K: p)$ are greater than 0.6. A track is identified as a pion if the likelihood ratios $\mathscr{L}(\pi: K)$ and $\mathscr{L}(\pi: p)$ are greater than 0.6. In addition, electron $\left(\mathscr{L}_{e}\right)$ likelihood is provided. A track with an electron likelihood greater than 0.95 is rejected. The efficiencies of hadron identification are about $90 \%$ for pions and kaons and $93 \%$ for protons. The $\pi^{0}$ candidates are selected from pair of photons whose invariant mass $\left(M_{\gamma \gamma}\right)$ satisfies $120<M_{\gamma \gamma}<150 \mathrm{MeV} / c^{2}$. The energy of each photon is required to be greater than $50 \mathrm{MeV} / \mathrm{c}^{2}$ and the energy of the $\pi^{0}$ candidate is required to be greater than $500 \mathrm{MeV} / \mathrm{c}^{2}$. The $\Lambda$ candidates are selected based on their decay vertex information [6] and invariant mass of a $\Lambda$ candidate is required to be within $3 \mathrm{MeV} / c^{2}$ of the nominal $\Lambda$ mass, which corresponds to approximately $3 \sigma$ of the mass resolution. The $K_{S}^{0}$ candidate is reconstructed from its decay into $\pi^{+} \pi^{-}$. The vertex of the two pions for the $K_{S}^{0}$ is required to be displaced from the interaction point (IP) in the direction of the pion pair momentum [7]. A pair of oppositely charged pions that have an invariant mass within $8 \mathrm{MeV} / c^{2}$ of the nominal $K_{S}^{0}$ mass, which corresponds to approximately $3.5 \sigma$ of the mass resolution, is selected. The $\Lambda_{c}^{+}\left(D^{+(0)}\right)$ candidates are selected by requiring invariant mass of the daughter particles to be within $1.5(2.0) \sigma$ of the nominal mass. The $\chi^{2}$ value of the common vertex fit of the $\Lambda_{c}^{+}$or $D^{(+) 0}$ is required to be less than 50 . For the remaining candidate, a mass constraint fit to the $\Lambda_{c}^{+}$or $D^{(+) 0}$ mass is performed to improve the momentum resolution. In order to reduce the combinatorial background, the scaled momentum $x_{p}=p^{*} / \sqrt{s / 4-m^{2}}$, where $p^{*}$ is the CM 
momentum of a $\Xi_{c}^{*}$ candidate and $s$ is CM energy squared and $m$ is mass of the $\Xi_{c}^{*}$ candidate, is required to be greater than 0.7 .

\section{Results}

\subsection{Results for $\Lambda_{c}^{+} K^{-} \pi^{+}$final state}

We select the $\Sigma_{c}(2455)^{++}\left(\Sigma_{c}(2520)^{++}\right)$region by requiring $\left|M\left(\Lambda_{c}^{+} \pi^{+}\right)-m_{\Sigma_{c}^{++}}\right|<5$ (18) $\mathrm{MeV} / c^{2}$, where $m_{\Sigma_{c}^{++}}$is the nominal mass of the $\Sigma_{c}(2455)^{++}$or $\Sigma_{c}(2520)^{++}$. Figure 1 (a) shows the $M\left(\Lambda_{c}^{+} K^{-} \pi^{+}\right)$distribution for the $\Sigma_{c}(2455)^{++}$signal region together with the same plot for the $\Sigma_{c}(2455)^{++}$sideband region. Clear peaks corresponding to the $\Xi_{c}(2980)^{+}, \Xi_{c}(3055)^{+}$and $\Xi_{c}(3080)^{+}$are seen. To obtain the statistical significance of the $\Xi_{c}(3055)^{+}$, an un-binned extended maximum likelihood (UML) fit is applied. PDFs for the $\Xi_{c}^{*}$ components are represented by a BreitWigner line-shape convolved with a Gaussian to account for the invariant-mass resolution. The background shape is assumed to be threshold function. To estimate the statistical significance of the $\Xi_{c}(3055)^{+}$, we evaluate the likelihood ratio $-2 \ln \left(\mathscr{L}_{0} / \mathscr{L}\right)$, where $\mathscr{L}_{0}$ is the likelihood for the fit without signal and $\mathscr{L}$ is likelihood for the fit with the signal taking into account the change of number of degrees of freedom. The statistical significance of the $\Xi_{c}(3055)^{+}$is $6.8 \sigma$.

Figure 1 (b) shows the $M\left(\Lambda_{c}^{+} K^{-} \pi^{+}\right)$distribution for the $\Sigma_{c}(2520)^{++}$selected region together with the same plot for the $\Sigma_{c}(2520)^{++}$sideband region. A clear peak corresponding to the $\Xi_{c}(3080)^{+}$is seen, while no peak structure is seen in the mass near $3.123 \mathrm{GeV} / c^{2}$. An UML fit is applied to extract the signal yield. Again, the $\Xi_{c}^{*}$ components are represented by a Breit-Wigner function convolved with a Gaussian. For the $\Xi_{c}(3080)^{+}$component, the mass and width of the Breit-Wigner are treated as free parameters; while for the $\Xi_{c}(3123)^{+}$component, the mass and width are fixed to the values obtained in Ref.[2]. The background shape is assumed to be threshold function. The yield of the $\Xi_{c}(3123)^{+}$is $8 \pm 22$ events, which is consistent with zero. Hence, a $95 \%$ C.L. upper limit for the product of the cross section and branching fraction of $\Lambda_{c}^{+}$produced with $x_{p}>0.7$ condition,

$$
\sigma_{\mathscr{B} \Lambda_{c}^{+}} \equiv \sigma\left(e^{+} e^{-} \rightarrow \Xi_{c}(3123)^{+} X\right) \times \mathscr{B}\left(\Lambda_{c}^{+} \rightarrow p K^{-} \pi^{+}\right)
$$

is evaluated with the Bayesian approach. As in Ref. [2], we assume $\mathscr{B}\left(\Xi_{c}(3123)^{+} \rightarrow \Sigma_{c}(2520)^{++} K^{-}\right)$ is equal to 1 . The upper limit on $\sigma_{\mathscr{B} \Lambda_{c}^{+}}$is $0.34 \mathrm{fb}$. The value is much smaller than that quoted in Ref. [2] $(1.6 \pm 0.6 \pm 0.2 \mathrm{fb})$. The systematic uncertainties of the masses and widths of the $\Xi_{c}^{*}$ and stability of the statistical significance of the $\Xi_{c}(3055)^{+}$are studied by changing various fit conditions. In none of these fitting configurations does the statistical significance of the $\Xi_{c}(3055)^{+}$fall below $6.6 \sigma$. The measured mass and width of the $\Xi_{c}^{*}$ states are summarized in Table 1 .

\subsection{Results for $\Lambda D^{+(0)}$ final state}

Figure 2 shows the $M\left(\Lambda D^{+(0)}\right)$ distribution, where peak structures near $3055 \mathrm{MeV} / c^{2}$ and $3080 \mathrm{MeV} / \mathrm{c}^{2}$ are seen. In order to check the existence of the peaking structure in the background, we check invariant mass distribution of the wrong-sign combination $\bar{\Lambda} D, \Lambda$ and $D$ for the sideband 

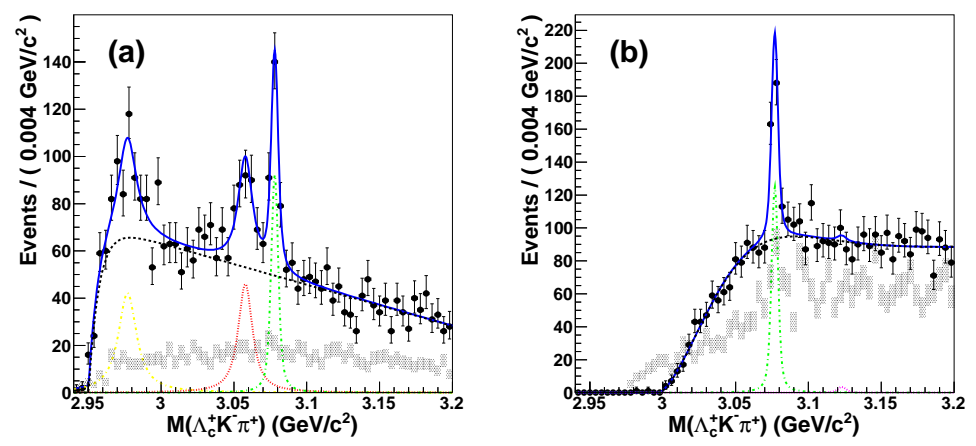

Figure 1: (a) The $M\left(\Lambda_{c}^{+} K^{-} \pi^{+}\right)$distribution with $\Sigma_{c}(2455)^{++}$selection. The dots with error bars show the distribution for the $\Sigma_{c}(2455)^{++}$selected whereas the rectangles show the distribution for the $\Sigma_{c}(2455)^{++}$ sideband region. Blue line shows the fit result. Black, yellow, red, and green lines show the contributions from the background, $\Xi_{c}(2980)^{+}, \Xi_{c}(3055)^{+}$, and $\Xi_{c}(3080)^{+}$, respectively. (b) The $M\left(\Lambda_{c}^{+} K^{-} \pi^{+}\right)$distribution with $\Sigma_{c}(2520)^{++}$selection. The dots with error bars show the distribution for $\Sigma_{c}(2520)^{++}$selected region whereas the rectangles show the distribution for the $\Sigma_{c}(2520)^{++}$sideband region. Blue line shows the fit result. Black, green, and purple lines show the contributions from the background, $\Xi_{c}(3080)^{+}$, and $\Xi_{c}(3123)^{+}$, respectively.

region the $\mathrm{D}$ candidates. None of them show a peaking structure. Therefore, these peaks are very likely to be corresponding to two known $\Xi_{c}^{*}$ states, $\Xi_{c}(3055)^{+}$and $\Xi_{c}(3080)^{+}$.

We perform UML fit to mass spectra again. PDFs for a $\Xi_{c}^{*}$ components are represented by Breit-Wigner line-shapes convolution with Gaussian. The mass and the width of the $\Xi_{c}^{*}$ states are treated as free parameters. The third order Chebychev function is used to model the combinatorial background shape. The statistical significances are obtained to be $11.9(4.7) \sigma$ for $\Xi_{c}(3055)^{+}$ $\left(\Xi_{c}(3080)^{+}\right)$and $7.6(2.6) \sigma$ for the $\Xi_{c}(3055)^{0}\left(\Xi_{c}(3080)^{0}\right)$. The systematic uncertainty of the mass and width are evaluated by changing various fit conditions. The measured mass and width of the $\Xi_{c}^{*}$ states are summarized in Table 1.
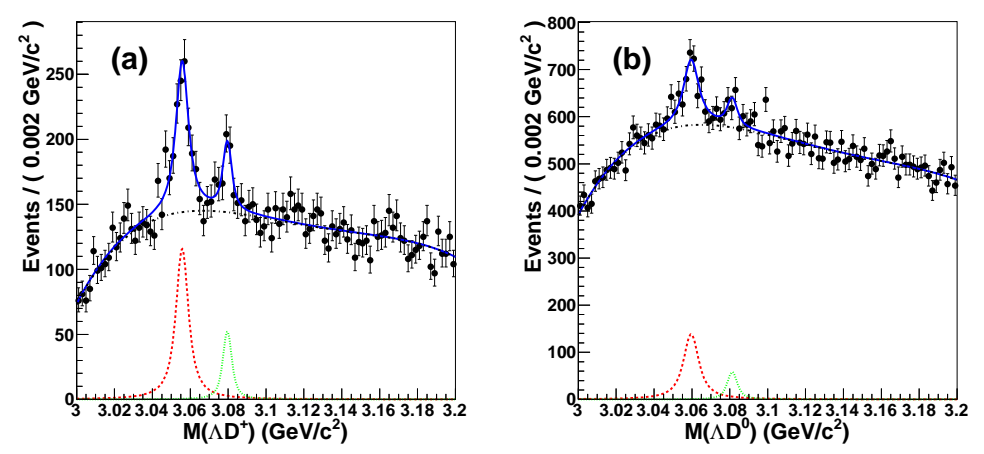

Figure 2: (a): $M\left(D^{+} \Lambda\right)$ distribution. (b): $M\left(D^{0} \Lambda\right)$ distribution. Blue line shows the fitting result. Black, red, and green lines show the background, $\Xi_{c}(3055)^{+/ 0}$, and $\Xi_{c}(3080)^{+/ 0}$ components, respectively. 
Table 1: The measured masses and widths of the $\Xi_{c}^{*+}$ states. The first error is statistical and second is systematic.

\begin{tabular}{l|ll}
\hline \hline Particle & Mass $\left(\mathrm{MeV} / c^{2}\right)$ & Width $(\mathrm{MeV})$ \\
\hline$\Xi_{c}(2980)^{+}$ & $2974.9 \pm 1.5 \pm 2.1$ & $14.8 \pm 2.5 \pm 4.1$ \\
$\Xi_{c}(3055)^{+}\left(\Sigma_{c}(2455)\right)$ & $3058.1 \pm 1.0 \pm 2.1$ & $9.7 \pm 3.4 \pm 3.3$ \\
$\Xi_{c}(3080)^{+}\left(\Sigma_{c}(2455)\right)$ & $3077.9 \pm 0.4 \pm 0.7$ & $3.2 \pm 1.3 \pm 1.3$ \\
$\Xi_{c}(3080)^{+}\left(\Sigma_{c}(2520)\right)$ & $3076.9 \pm 0.3 \pm 0.2$ & $2.4 \pm 0.9 \pm 1.6$ \\
$\Xi_{c}(3055)^{+}\left(\Lambda D^{+}\right)$ & $3055.7 \pm 0.4 \pm 0.4$ & $7.1 \pm 1.2 \pm 1.8$ \\
$\Xi_{c}(3080)^{+}\left(\Lambda D^{+}\right)$ & $3079.6 \pm 0.6 \pm 0.7$ & $4.0 \pm 1.5 \pm 1.0$ \\
$\Xi_{c}(3055)^{0}\left(\Lambda D^{0}\right)$ & $3059.7 \pm 0.6 \pm 0.5$ & $7.4 \pm 1.9 \pm 3.4$ \\
$\Xi_{c}(3080)^{0}\left(\Lambda D^{0}\right)$ & $3081.6 \pm 1.1 \pm 0.2$ & $4.4 \pm 1.8 \pm 1.9$ \\
\hline \hline
\end{tabular}

\section{Summary}

We report studies of charmed strange baryons in the $\Lambda_{c}^{+} K^{-} \pi^{+}$and $\Lambda D^{+(0)}$ final states. We have searched for the $\Xi_{c}(3055)^{+}$and $\Xi_{c}(3123)^{+}$in the $\Lambda_{c}^{+} K^{-} \pi^{+}$decays through intermediate $\Sigma_{c}(2455)^{++}$or $\Sigma_{c}(2520)^{++}$states. We observe the $\Xi_{c}(3055)^{+}$while we do not observe any significant signal corresponding to the $\Xi_{c}(3123)^{+}$. We also report first observation of $\Xi_{c}(3055)^{+(0)}$ and $\Xi_{c}(3080)^{+}$decay in the $\Lambda D^{+(0)}$ final states. Especially, this is the first observation of the $\Xi_{c}(3055)^{0}$.

\section{References}

[1] R. Chistov et al. [Belle Collaboration], Phys. Rev. Lett. 97, 162001 (2006) [hep-ex/0606051].

[2] B. Aubert et al. [BaBar Collaboration], Phys. Rev. D 77, 012002 (2008) [arXiv:0710.5763 [hep-ex]].

[3] A. Abashian et al. (Belle Collab.), Nucl. Instr. and Meth. A 479, 117 (2002); also see detector section in J.Brodzicka et al., Prog. Theory. Exp. Phys. (2012) 04D001.

[4] S. Kurokawa and E. Kikutani, Nucl. Instr. and. Meth. A499, 1 (2003), and other papers included in this volume; T.Abe et al., Prog. Theor. Exp. Phys. (2013) 03A001 and following articles up to $03 \mathrm{~A} 011$.

[5] Throughout this paper, the inclusion of the charge-conjugate decay mode is implied unless otherwise stated.

[6] K. Abe et al. [Belle Collaboration], Phys. Rev. D 65, 091103 (2002) [hep-ex/0203027].

[7] K. Sumisawa et al. [Belle Collaboration], Phys. Rev. Lett. 95, 061801 (2005). 